\title{
lid Swelling: A Window to Diagnosis of Sarcoidiosis
}

\author{
R.C. Gupta, R.N. Kushwaha, Priyanka Gupta, Parul Singh \\ From the Department of Ophthalmology, GSVM Medical \\ College, Kanpur, Uttar Pradesh, India.
}

\section{Abstract:}

Sarcoidosis is a systemic inflammatory disease which commonly involves lungs, lymph nodes, skin and eyes. We have come across a 55 year male who presented with painless, progressively increasing swelling at medial aspect of right upper and lower eyelids since 8 months. There were no other ocular or systemic complaints. The patient was a known case of controlled diabetes mellitus. Swellings were soft, non-tender, non-fluctuant and not fixed to underlying structures. Overlying skin was normal, freely mobile. Rest ocular examination was normal. Excisional biopsy with clear margins was planned. Histopathological examination report showed sarcoidosis. On two year follow-up patient developed no other ocular, systemic or laboratory signs of systemic sarcoidosis. This case highlights that even isolated lid swellings can be a pointer to diagnosis of sarcoidosis and can be the only clinical presentation.

Key words: Sarcoidosis, Lymph Nodes, Eye, Eyelids, Edema, Diabetes Mellitus.

\section{Introduction}

Sarcoidosis is a systemic inflammatory disease characterized by the formation of non caseating granulomas in affected organs. The disease was first described in 1878 by noted surgeon, Sir Jonathan Hutchinson as a dermatologic disorder [1].

The disease most commonly involves lungs, lymph nodes, skin, and eyes. Ocular involvement occurs in approximately $25 \%$ of patients with sarcoidosis. The uveal tract is the most common site of ocular involvement followed by conjunctiva and lacrimal gland. Ocular sarcoidosis can manifest itself with blurred vision, photophobia, floaters, redness, pain or systemic features depending on organ and extent of involvement. But we have come across a rare presentation with isolated lid involvement, as reported by Martins MC et al [2].

\section{Case Report}

A 55 year male presented to our outpatient department with painless and progressive increasing swelling at medial aspect of right upper and lower eyelids since 8 months [Fig.1]. There were no other ocular or systemic

Corresponding Author: Dr. Priyanka Gupta

Email: priyankagupta8405@gmail.com

Received: April 29, 2013 | Accepted: May 6, 2013 | Published Online: May 25, 2013

This is an Open Access article distributed under the terms of the Creative Commons Attribution License (creativecommons.org/licenses/by/3.0)

Conflict of interest: None declared | Source of funding: Nil | DOl: http://dx.doi.org/10.17659/01.2013.0036 
complaints. The patient was a known case of controlled diabetes mellitus.

On examination best corrected visual acuity of both eyes was $20 / 20$. Two swellings of $1 \times 1 \mathrm{~cm}$ each were present on medial aspect of right upper and lower lid [Fig.1]. They were soft, non-tender, non-fluctuant, not fixed to underlying structures. Overlying skin was normal and freely mobile. Swellings were not associated with difficulty in eyelid closure or lid to globe apposition. Lacrimal apparatus examination was normal. Ocular movements were normal in all gazes. Rest of the anterior segment was normal on slit lamp examination. Fundus examination on direct and indirect ophthalmoscopy showed no abnormality. Diagnostic dilemma led to excisional biopsy with clear margins under local anaesthesia. Histopathological examination report showed sarcoidosis [Fig.2].

A comprehensive ocular and systemic evaluation was carried out with collaboration of physicians. Interestingly neither any other ocular lesion nor any systemic involvement was found. The tissue block was reviewed for confirmation of the diagnosis.

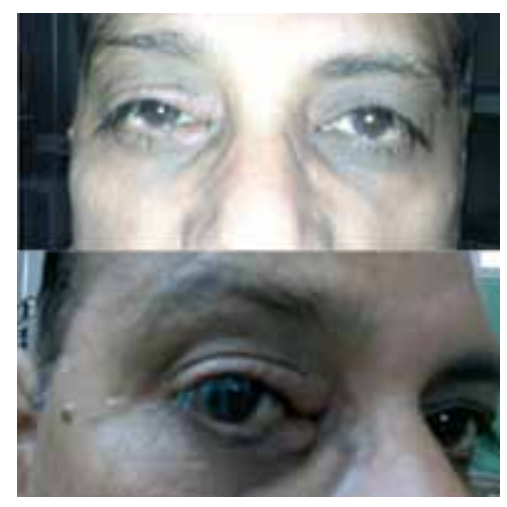

Fig.1: Swelling over right upper and lower eyelid.

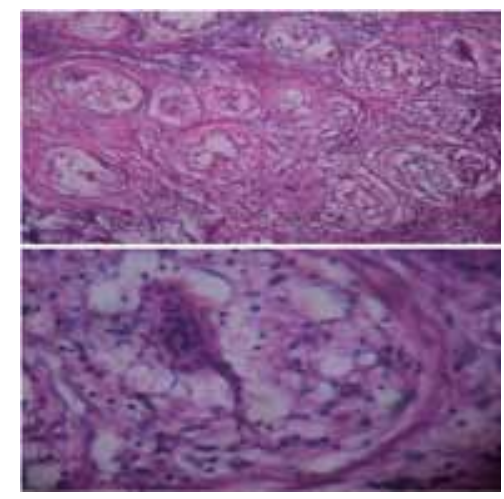

Fig.2: Histopathological slide suggesting sarcoidosis. 
The patient was followed up for next two year but he developed no other ocular or systemic signs of sarcoidosis. The laboratory tests and imaging investigations showed no signs of systemic sarcoidosis. The diagnosis of ocular sarcoidosis was made retrospectively on basis of histopathology after excluding all other possibilities.

\section{Discussion}

Sarcoidosis is a systemic inflammatory non-caseating granulomatous disease involving lungs, lymph nodes, skin and other organs. Ocular involvement occurs in approximately $25 \%$ of patients with sarcoidosis. The uveal tract is the most common site of involvement in ocular sarcoidosis. Of all cases of uveitis, sarcoidosis accounts for $3-10 \%$ of cases. Uveitis can also precede pulmonary symptoms by several years. Of all sarcoid uveitis cases, anterior uveitis occurs in $22-70 \%$ of patients while $28 \%$ of patients have posterior uveitis. Sarcoidosis involves the conjunctiva in 6-40\% of cases [3-5] and lacrimal gland in 15-28\% [6]. Although the facial nerve is the most common cranial nerve affected but 3rd, 4th, 6th cranial nerves along with extra-ocular muscles are also commonly involved $[7,8]$. Cataracts have been reported in $8-17 \%$ of patients. Corneal involvement in form of inferior corneal thickening, calcific band keratopathy, stromal thinning and interstitial keratitis is seen [9]. Orbital and eyelid granulomas have been reported. Optic neuropathy and glaucoma have been associated though rare. Scleral involvement is rare in sarcoidosis. In our case the anterior segment was normal on detailed examination.

Posterior segment shows findings like snowball or string of pearls vitreous opacity, vascular occlusion, cystoid macular edema, retinal perivasculitis, retinochoroidal patchy exudates, retinal choroidal exudates (candle wax appearance), choroidal nodule, neovascularization of disc and retina. No abnormalities were found in fundus on detailed examination and investigations.

Two classic syndromes are associated with ocular involvement. Lo" fgren's syndrome is the association of erythema nodosum and bilateral hilar lymphadenopathy (BHL) due to sarcoidosis. Arthritis and iritis may be present [10]. Heerfordt's syndrome is characterised by kerotconjunctivitis sicca, parotid dysfunction with swelling, facial nerve paralysis and lacrimal gland dysfunction [11,12]. Granulomas can also be seen on the liver, spleen, salivary glands, heart, bones and nervous system.

But our case was very atypical as there were no systemic or ocular manifestations other than lid swellings and diagnosis was made retrospectively on basis of histopathology after excluding all other possibilities. Other similar case was reported by Martins MC et al [2].

\section{Conclusion}

This case highlights that even isolated lid swelling can be a pointer to diagnosis of sarcoidosis and can be the only clinical presentation. Therefore, sarcoidosis should also be considered in differential diagnosis of lid swelling.

Conflict of interest: None stated. 


\section{References}

1. Young, Roscoe C, Rachal, Raylinda E, Cowan, Claude L. Sarcoidosis - The Beginning: Historical Highlights of Personalities and Their Accomplishments During the Early Years. Journal of the National Medical Association. 1984;9:887-896.

2. Martins MC, Pessoa de Souza Filho J, Sant'Anna AE, Coutinho AB, Burnier MN Jr, Rigueiro MP. Eyelid swelling as the only manifestation of ocular sarcoidosis. Ocul Immunol Inflamm. 2005;13:399-402.

3. Khan F, Wessley Z, Chazin SR, Seriff NS. Conjuctival biopsy in sarcoidosis. Annals of Opthalmology. 1977;9:671-676.

4. Smith JA, Foster CS. Sarcoidosis and its ocular manifestations. Internat Ophthalmol Clin. 1996;36:109125.

5. Spaide RF, Ward DL. Conjunctival biopsy in the diagnosis of sarcoidosis. Br J Ophthalmol. 1990;74:469471.

6. Jabs DA, Johns CA. Ocular involvement in chronic sarcoidosis. Am J Opthalmol. 1986;102:297-301.

7. Lower EE, Broderick JP, Brott TG, Baughman RP. Diagnosis and management of neurologic sarcoidosis. Arch Intern Med. 1997;157:1864-1868.

8. Zajicak JP, Scolding NJ, Foster O. Central nervous system sarcoidosis - diagnosis and management. Q J Med. 1999;92:103-117.

9. Mayers M. Ocular sarcoidosis. Int Ophthalmol Clin. 1990;30:257-263.

10. Mana J, Gomez Vaquero C, Montero A. Lofgren's syndrome revisited: a study of 186 patients. Am J Med. 1999;107:240-245.

11. Glocker FX, Seifert C, Lucking CH. Facial palsy in heerfordt's syndrome: Electrophysiological localization of the lesion. Muscle Nerve. 1999;22:1279-1 282.

12. James DG, Sharma OP. Parotid gland sarcoidosis. Sarcoidosis Vasc Diffuse Lung Dis. 2000; 17:27-32. 